face so as to physically limit the number of metal atoms that could join together in each cluster.

While measuring the properties of the clusters, the researchers found that, in one cluster, the circular dichroic effect exceeded $300 \mathrm{ppm}$ in the yellow-green region. In another cluster, the effect exceeded 1000 ppm in the red and near-infrared. As reported in the researchers' article in the March 30 issue of the Journal of Physical Chemistry, these optical measurements suggest that the clusters have a helical structure.

Schaaff said, "Such effects had not previously been measured in metal-cluster compounds, and it's kind of a shock that small metals might prefer to have a helical structure."

Using gel electrophoresis to separate the clusters by weight, Schaaff found that certain cluster sizes dominated, with 28-atom assemblies-slightly less than $1 \mathrm{~nm}$ across-being the most common. The chiral properties varied by the size of the cluster and, therefore, were only observed clearly when the clusters were separated by weight.

Only clusters with 40 or fewer atoms displayed the intense optical properties. The optical effect changed direction as the researchers moved from one cluster size to the next, suggesting a direct correlation to the energies of the conduction electrons in the metal's outer shell.

Whetten, a professor in the School of Physics and School of Chemistry and Biochemistry, said, "Even though the optical absorption increases more or less monotonically here, the preferences for right- versus left-handed light changes direction from one band to another." He said that the effect may be related to the high level of confinement created in the conduction electrons by formation of the small clusters, but research has not yet confirmed this. A helical geometrical pattern or "tiling" of the glutathione adsorption sites (gold-sulfur bonds) could also affect the circulation of the conduction electrons, he said.

\section{Feedback-Controlled Lithography Enables Molecule Manipulation on Atomic Scale}

Researchers at the University of Illinois-Urbana-Champaign have tethered individual organic molecules at specific locations on silicon surfaces. Joseph Lyding, a professor of electrical and computer engineering and a researcher at the university's Beckman Institute for Advanced Science and Technology, and his research team first passivated the silicon bonds with hydrogen. They then used an ultrahigh vacuum scanning tunneling microscope to break individual siliconhydrogen bonds and dislodge hydrogen atoms from selected sites.

Graduate student Mark Hersam said, "By removing individual hydrogen atoms, we create holes in the clean silicon surface. Since these holes-or dangling bonds-serve as effective binding sites, molecules injected in the gas phase will spontaneously self-assemble into the predefined patterns."

A technique called feedback-controlled lithography gives the patterning process an atomic precision. "Feedback-controlled lithography works by actively monitoring the microscope feedback signal and the tunneling current during patterning, and immediately terminating the patterning process when a bond is broken," Lyding said. "By operating the microscope under feedback control, a carefully controlled dose of electrons can be written along a line or over an area to locally depassivate the surface and create templates of individual dangling bonds."

As reported in the June issue of Nanotechnology, the researchers demonstrated the feasibility of their technique with three organic molecules: norbornadiene, copper phthalocyanine, and carbon- 60 buckyballs. The researchers said that one advantage of organic molecules is that their end groups can be functionalized for potential electronic or mechanical switching properties.

Hersam said that while the technology for economically tethering billions of molecular devices on a chip surface does not yet exist, they can fabricate small numbers of the devices and test their function. He said, "This is an important step in bridging the gap between molecular electronics and silicon technology."

\section{Single-Isotope Silicon Helps Chips Keep Their Cool}

In Vol. 15 of Solid State Communications, a research group from the Max Planck Institute for Solid State Research in Stuttgart reports a substantial enhancement of the thermal conductivity in a bulk silicon crystal made from only one isotope $\left({ }^{28} \mathrm{Si}\right)$. At room temperature, singleisotope silicon (SISSI) is a $60 \%$ better heat conductor than natural silicon, which consists of three stable isotopes: $92.2 \%$ ${ }^{28} \mathrm{Si}, 4.7 \%{ }^{29} \mathrm{Si}$, and $3.1 \%{ }^{30} \mathrm{Si}$. At $77 \mathrm{~K}$, the temperature of liquid nitrogen, the enhancement amounts to a factor of 2.4, while at $20 \mathrm{~K}$, where the thermal conductivity has its maximum, it is close to a factor of 6 . The maximum thermal conductivity of SISSI $\left(30,000 \mathrm{Wm}^{-1} \mathrm{~K}^{-1}\right)$ exceeds that of the best natural heat conductor, namely diamond, by a factor of 2.5 .
The thermal conductivity enhancement in SISSI is based on the fact that phonons, the quantized lattice vibrations, are scattered by fluctuations of their atomic masses, as present in most conventional crystals, which contain a mixture of different stable isotopes. The suppression of this scattering in isotopically enriched crystals results in an enhancement of the thermal conductivity that can be strong. For example, the removal of about $8 \%$ of the "isotopic impurities" (present in natural silicon) in the nominally isotopically pure SISSI crystal (enriched to $99.8588 \%$ ${ }^{28} \mathrm{Si}$ ) investigated by the researchers is sufficient to increase the thermal conductivity by almost an order of magnitude. The thermal-conductivity enhancement varies with temperature due to the presence of other phonon-scattering mechanisms, such as boundary scattering (at low temperatures) and "umklapp" scattering (at high temperatures).

\section{Hysteresis and Spikes Observed in Quantum Hall Effect}

Researchers at Columbia University and Bell Laboratories have recently observed strong hysteresis and the formation of sharp peaks in the integral quantum Hall effect (IQHE) in magneto transport experiments on two-dimensional electron gas (2DEG) at $\sim 0.1 \mathrm{~K}$. The researchers used 300$\AA$-wide quantum-well structures of modulation-doped GaAs/AlGaAs, grown by molecular-beam epitaxy (MBE). The well is $\delta$-doped on both sides with silicon impurities at a distance of $950 \AA$ and $750 \AA$ for two different types of samples; the density is 2.3 $\times 10^{11} \mathrm{~cm}^{-2}$ and $3.2 \times 10^{11} \mathrm{~cm}^{-2}$, respectively. The researchers performed transport measurements using standard lock-in techniques in a dilution refrigerator with a base temperature of $70 \mathrm{mK}$. The results are similar for both ac and dc current.

The hysteretic spikes on the magnetoresistance $R_{x x}$ are most pronounced at integral filling factors $v$ between 3 and 12, and the details of the hysteresis vary among the peaks. The time scale of the peak decay is in the range of minutes to several hours, which is enormously long for 2DEG resistance decay. Momentary grounding of the contacts, however, causes a dramatic decrease in the amplitude of the peaks, suggesting the presence of a nonuniform, nonequilibrium configuration in the specimen. In addition, the hysteresis and peaks disappear if a negative voltage bias is applied to the gate at the back of the sample.

The researchers conclude, in the May 15 issue of Physical Review B, that there exists a parallel conducting path in the form of a low-density, disordered, two- 
dimensional impurity channel (2DIC) on the bottom side of the quantum well. The peaks observed in the IQHE regime of the 2DEG reflect the resistivity of the 2DIC $\left(\rho_{x x}^{2 D I C}\right)$. In that regime, the coupling between the two channels leads to a nonequilibrium charge distribution in the 2DEG with very long RC characteristic times, which causes the peak phenomena to last for a long time. The influence of the 2DEG nonequilibrium charge distribution on the 2DIC resistivity pattern is observed as the hysteretic peaks. The density of 2 DIC, estimated at about $5.7 \times 10^{10} \mathrm{~cm}^{-2}$, can drop below the conducting limit when a negative bias is applied to the back-side gate, causing the hysteresis to disappear.

WIRAWAN PURWANTO

\section{Lattice-Dynamics Properties May Explain Low Dielectric Constant of Thin Films}

Soft-mode phonons are known to play a key role in the physics of ferroelectrics. The softening of one particular phonon usually results in a dramatic increase in the dielectric constant and an appearance of the ferroelectric phase. The relative variation of the soft-mode frequency with the dielectric constant is fully described by the Lyddane-Sachs-Teller (LST) formula.

Researchers from The Pennsylvania State University and Max Planck Institute for Solid State Research have reported in the March 23 issue of Nature the relationship between the lower dielectric constant of thin films and the reduced softening of the lowest optical-phonon mode. This research may explain the mechanism of low dielectric constant in thin films.

After applying the dead-layer modelwhich takes into account interface effects and results in a decrease of the average dielectric constant-to derive a temperature-dependent dielectric constant for thin $\mathrm{SrTiO}_{3}$ (STO) films, the researchers found that the model was not able to accurately describe the measured dielectric constants. According to the researchers, latticedynamics studies are necessary for a better explanation of the lower dielectric constant of thin films.

The researchers used pulsed laser deposition to prepare the samples: They heated the substrate to $720^{\circ} \mathrm{C}$ in an oxygen pressure of $100 \mathrm{mTorr}$, then cooled the as-deposited film to room temperature in 400 Torr of oxygen. They then deposited STO films with thicknesses of $0.5,1$, and $2 \mu \mathrm{m}$ on a $0.35-\mu \mathrm{m}$ conducting oxide $\mathrm{SrRuO}_{3}$ buffer layer.

Although the researchers observed optical-phonon peaks in STO films by means of conventional Raman scattering, the soft mode (the lowest optical mode) was not detected in this way. For this reason, they carried out far-infrared ellipsometry measurements at the National Synchrotron Light Source. Taking advantage of the high brightness of the synchrotron and Fourier transform infrared (FTIR) spectroscopy, the complex dielectric functions had been directly measured in the frequency range of $30-700 \mathrm{~cm}^{-1}$ and at a temperature range of $5-300 \mathrm{~K}$.

From the plots of the real and imaginary parts of the measured complex dielectric constants versus wave numbers, the soft-mode optical TO1 (transverse) phonon has been clearly observed, as well as other TO modes. It proved the feasibility of the experimental method.

In order to acquire the frequency of the soft mode, the researchers fit the experimental spectra to the dielectric function that takes into account the contribution of the phonon broadening. They found that the eigenfrequency of the soft mode (TO1) decreases as the temperature is lowered and saturates at $13 \mathrm{~cm}^{-1}$ instead of $62 \mathrm{~cm}^{-1}$ for the bulk crystal. On the other hand, similar eigenfrequencies of other longitudinal (LO) and TO modes have been observed both in the thin film and the bulk.

By comparing the results with the LST formula, the researchers observed the correlation between the harder soft mode in the thin film and a lower static dielectric constant. The LST relation between the optical-phonon eigenfrequencies and the static dielectric constant has been found to be fully maintained in thin films like $\mathrm{SrTiO}_{3}$ as well as in the bulk material. The researchers further said that "besides the dead-layer effect, the lower dielectric constant in STO thin films is due to their fundamental lattice dynamical propertiesspecifically, the soft-mode hardening."

In their discussion of the physical mechanisms for the soft-mode hardening, the researchers found that local strain and defects play key roles.

JIDONG HOU

\section{Chiral Diarylethene Dopant Induces Large Photostimulated Pitch Change in Nematic Liquid Crystals}

In an effort to explore photostimulated reversible phase changes in liquid crystals, researchers at Osaka Prefecture University, Kyushu University, and CREST have developed a new photoactive chiral chromophore-that is, a chiral cyclohexane containing two diarylethenes. In the April issue of Chemistry of Materials, the researchers refer to this compound as com- pound 1. They explored the photoisomerization of compound $\mathbf{1}$ and its influence on the properties of a doped nematic liquid crystal and observed a large and stable photostimulated pitch change in a chiral nematic phase induced by this compound.

With UV light irradiation (a mercury lamp, $254 \mathrm{~nm}, 10 \mathrm{~W}$ ), the researchers converted open-ring isomers of compound 1 to stable closed-ring isomers. Irradiated by visible light (a halogen lamp, $\lambda>480 \mathrm{~nm}$, $100 \mathrm{~W}$ ), the closed-ring isomers were switched back to open-ring isomers. The researchers doped a $1.0 \mathrm{wt} \%$ open-ring isomer of compound 1 into a nematic liquidcrystalline material, 4-cyano- $4^{\prime}$-pentylbiphenyl (K-15), at $26^{\circ} \mathrm{C}$ and achieved an apparent nematic phase. An induced cholesteric phase (chiral nematic phase) resulted at content higher than $5.0 \mathrm{wt} \%$. When the doped liquid crystal $(1.0 \mathrm{wt} \%)$ was irradiated with UV light for $3 \mathrm{~min}$, the original stable nematic texture turned into a cholesteric fingerprint pattern (observed by a polarization microscope) and the pitch of the liquid crystalline phase decreased from longer than $40 \mu \mathrm{m}$ to $11.8 \mu \mathrm{m}$ (measured by the droplet method). When irradiated with visible light for another $3 \mathrm{~min}$, the observed texture gradually returned to the stable nematic texture, and the measured pitch went back to longer than $40 \mu \mathrm{m}$. This switching cycle was performed more than 50 times without deterioration of the liquid-crystalline phase. The researchers said that the twisting power of the closedring isomers is much larger than that of the open-ring isomers and this accounts for the above photoresponsive behavior.

In addition, the researchers prepared an azobenzene derivative and compared its optical rotation value with that of compound 1 . They conclude that the large increase in the optical rotation value of compound 1 plays a role in the high efficiency of the phase change.

ZHENGMAO ZHU

\section{Intraband Electron Energy Relaxation in CdSe Quantum Dots is Dominated by Coulombic Coupling Between Electrons and Holes}

Femtosecond (fs) transient absorption measurements of CdSe quantum dots capped with either a ZnS layer or a pyradine layer indicate that electron intraband energy relaxation is dominated by Coulombic electron-hole coupling effects rather than by phonon-mediated processes (as is true of bulk materials) or Augerassisted processes (i. e., electron-hole recombination with the excess energy given to a hole). Researchers at Los 